\title{
Gambogic Acid Lysinate Induces Apoptosis in Breast Cancer MCF-7 Cells by Increasing Reactive Oxygen Species
}

\author{
Yong-Zhan Zhen, ${ }^{1}$ Ya-Jun Lin, ${ }^{2}$ Kai-Ji Li, ${ }^{1}$ Xiao-Shan Yang, ${ }^{2}$ Yu-Fang Zhao, \\ Jie Wei, ${ }^{2}$ Jing-Bo Wei, ${ }^{1}$ and Gang $\mathrm{Hu}^{2}$ \\ ${ }^{1}$ Department of Histology and Embryology, Basic Medical College of Hebei United University, Tangshan 063000, China \\ ${ }^{2}$ The Key Laboratory of Geriatrics, Beijing Hospital \& Beijing Institute of Geriatrics, Ministry of Health, No. 1 Dahua Road, \\ Dongdan, Dongcheng District, Beijing 100730, China
}

Correspondence should be addressed to Gang Hu; huganglys2010@126.com

Received 8 August 2014; Revised 2 December 2014; Accepted 15 December 2014

Academic Editor: Senthamil R. Selvan

Copyright (c) 2015 Yong-Zhan Zhen et al. This is an open access article distributed under the Creative Commons Attribution License, which permits unrestricted use, distribution, and reproduction in any medium, provided the original work is properly cited.

Gambogic acid (GA) inhibits the proliferation of various human cancer cells. However, because of its water insolubility, the antitumor efficacy of GA is limited. Objectives. To investigate the antitumor activity of gambogic acid lysinate (GAL) and its mechanism. Methods. Inhibition of cell proliferation was determined by MTT assay; intracellular ROS level was detected by staining cells with DCFH-DA; cell apoptosis was determined by flow cytometer and the mechanism of GAL was investigated by Western blot. Results. GAL inhibited the proliferation of MCF-7 cells with $\mathrm{IC}_{50}$ values $1.46 \mu \mathrm{mol} / \mathrm{L}$ comparable with GA (IC ${ }_{50}$, $1.16 \mu \mathrm{mol} / \mathrm{L})$. GAL promoted the production of ROS; however NAC could remove ROS and block the effect of GAL. GAL inhibited the expression of SIRT1 but increased the phosphorylation of FOXO3a and the expression of p27Kip1. At knockdown of FOXO3a, cell apoptosis induced by GAL can be partly blocked. In addition it also enhanced the cleavage of caspase-3. Conclusions. GAL inhibited MCF-7 cell proliferation and induced MCF-7 cell apoptosis by increasing ROS level which could induce cell apoptosis by both SIRT1/FOXO3a/p27Kip1 and caspase-3 signal pathway. These results suggested that GAL might be useful as a modulation agent in cancer chemotherapy.

\section{Introduction}

Breast cancer represents the most common cancer in women in western countries. One in 8 women in the United States will develop breast cancer in her lifetime, while its incidence rate constantly increases in developing countries [1, 2]. Over the past decade, although the introduction of new drugs, including paclitaxel and Herceptin, has improved the treatment landscape for breast cancer patients, some patients continue to experience drug resistance and disease relapse $[3,4]$. Therefore, further investigations to find a novel antibreast-cancer drug should be conducted.

Gambogic acid (GA) is the principal active component of gamboge, the resin from various Garcinia species including G. Morella and G. hanburyi [5]. It was reported in traditional Chinese medical documents that GA possessed diverse biological effects such as anti-inflammatory and antipsoriatic efficacy [6, 7], anti-invasive effect [8], inhibiting angiogenesis [9], and inducing cell apoptosis [10]. One of the major barriers for GA clinical application is its insolubility in water. GA could only dissolve in DMSO [11], and DMSO is rather harmful to cells. Even though it dissolves in DMSO, if diluted by water, it will precipitate. Thus it impedes the uses of GA. In the present study, we prepared gambogic acid lysinate (GAL), which can dissolve in water, and the solubility of GAL in water is $1.16 \mathrm{~g}$. No report about the manufacture of GAL and its antitumor activity has been found yet. In this study, we investigated the antitumor activity of GAL.

\section{Materials and Methods}

2.1. Chemicals and Antibodies. Gambogic acid (98\%) was purchased from Nanjing Jingzhu Bio-technology Ltd. (Nanjing, Jiangsu, China). Lysine was purchased from Beijing 


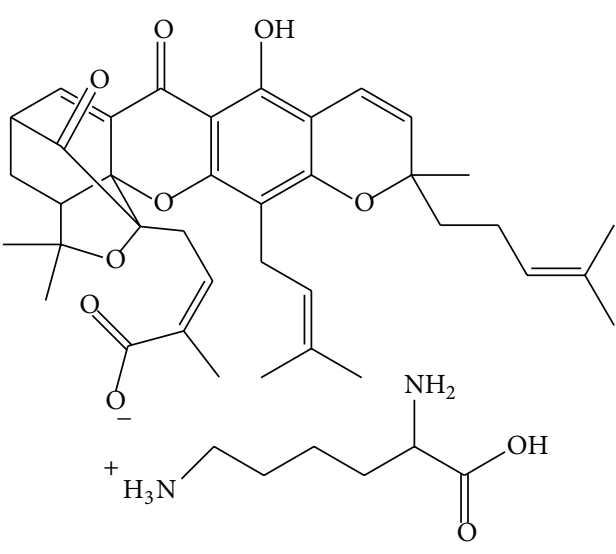

(a)

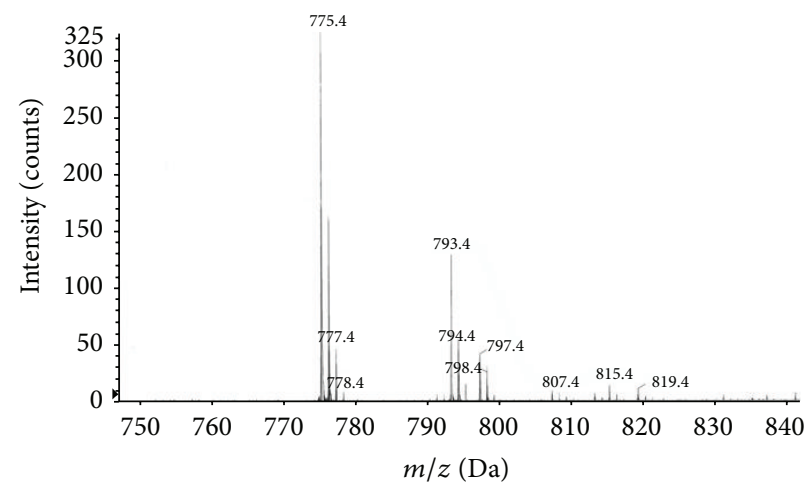

(b)

FIGURE 1: The structure and the determination of gambogic acid lysinate. Gambogic acid and lysine combine together through ionic bond. It is determined by Matrix-Assisted Laser Desorption/Ionization Time of Flight Mass Spectrometry and the peak value (755.4 Da) is gambogic acid lysinate.

Solarbio Science and Technology Co. (Beijing, China). Gambogic acid lysinate (GAL) was made in our department. 3-(4,5-Dimethylthiazol-2-yl)-2,5-diphenyltetrazolium bromide (MTT) and dimethyl sulfoxide (DMSO) were obtained from Sigma Aldrich (Shanghai, China). FOXO3a siRNA and the scrambled siRNA (NC siRNA) control were supplied by Santa Cruz Technology (Dallas, TX, USA). Antibodies against SIRT1, FOXO3a, p-FOXO3a (s294), p27Kip1, caspase-3 and cleaved-caspase-3 (C-caspase-3) were purchased from Cell Signaling Technology (Beverly, MA, USA). Antibody against $\beta$-actin was purchased from Santa Cruz Technology (Dallas, TX, USA). Secondary antibodies were purchased from Cell Signaling Technology.

2.2. The Protocol of GAL Preparation and Matrix-Assisted Laser Desorption/Ionization Time of Flight Mass Spectrometry (MALDI-TOF MS) Analysis. Lysine (29.2 mg) was dissolved in distilled water and then gambogic acid $(62.9 \mathrm{mg})$ was added to lysine solution. After lysine reacted with gambogic acid at $30^{\circ} \mathrm{C}$ for 24 hours, the solution was frozen and dried in freeze-drier (SCIENTZ, Ningbo, China) for 12 hours. An Agilent 6500 MS system (Agilent Technologies, CA, USA) was used to analyze the molecular weight of new product. The analysis method is following. Ionization is achieved using electrospray in the positive mode with the spray voltage set at $4.0 \mathrm{kV}$. The fragmentor voltage and collision energy were optimized during tuning as $150,18 \mathrm{eV}$ for GAL. Analysis was carried out in electrospray positive ionization using multiple reaction monitoring modes. The mass transition ion pair was selected as $m / z \quad 840 \rightarrow 750$ for GAL. The ion spectra of product are represented in Figure 1(b). The data acquisition software used was MassHunter software (Agilent Corporation, MA, USA).

2.3. Cell Culture and Cytotoxicity Assay. Human breast cancer cell lines MCF-7 were used in this study. MCF-7 cells were maintained in DMEM medium supplemented with
$10 \%$ fetal bovine serum (Hyclone, Logan, UT, USA). Cells were grown in a humidified atmosphere of $5 \% \mathrm{CO}_{2}$ at $37^{\circ} \mathrm{C}$. In vitro cytotoxicity of GAL was determined using 3(4,5-dimethylthiazol-2-yl)-2,5-diphenyltetrazolium bromide (MTT) assay. Briefly, MCF-7 cells were transferred to 96-well tissue culture plates at a density of $3 \times 10^{3}$ cells per well, 24 hours prior to treatment. The medium was then replaced with fresh medium containing GAL at different concentrations. The culture medium without any drug formulation was used as the control. After 48 hours of incubation, medium was removed and cells were washed once with sterile phosphate buffered saline (PBS). Then $20 \mu \mathrm{L}$ of MTT solution $(5 \mathrm{mg} / \mathrm{mL})$ was added to each well and further incubated for 4 hours. Medium was removed and $150 \mu \mathrm{L}$ DMSO was added to each well to dissolve the purple formazan crystal converted from MTT. Optical density at $570 \mathrm{~nm}$ was determined with a SpectraMax 190 Absorbance Microplate Reader (Sunnyvale, CA, USA) and the concentration at which $50 \%$ of growth is inhibited $\left(\mathrm{IC}_{50}\right)$ was calculated by GraphPad Prism 5.0 (GraphPad, La Jolla, CA, USA).

2.4. Examination of Intracellular Reactive Oxygen Species (ROS) Accumulation. Intracellular hydrogen peroxide levels were monitored by fluorescence microscopy and fluorescence spectrophotometer after staining with DCFH-DA (dichlorodihydro-fluorescein diacetate; Molecular Probes, Eugene, OR, USA). Briefly, cells in a logarithmic growth phase $(2 \times$ $10^{5}$ cells per well in a $25 \mathrm{~mm}^{2}$ polystyrene culture flask) were treated with GAL for $24 \mathrm{~h}$ and and then labeled with $10 \mu \mathrm{mol} / \mathrm{L}$ DCFH-DA for $1 \mathrm{~h}$. Next, the cells were monitored by Olympus inverted fluorescence microscope (Tokyo, Japan) and fluorescence spectrophotometer (Cary Eclipse, Palo Alto, CA, USA). The percentage of cells displaying increased dye uptake was used to reflect an increase in ROS levels.

2.5. Hoechst 33258 Staining. The nuclear fragmentation in MCF-7 cells treated with different concentrations of GAL 
TABLE 1: The apoptosis ratio of different groups.

\begin{tabular}{lcccc}
\hline Groups & Normal (\%) & Necrosis (\%) & Early apoptosis (\%) & Late apoptosis (\%) \\
\hline Control & 96.20 & 0.50 & 1.00 & 2.30 \\
GAL $0.5 \mu \mathrm{mol} / \mathrm{L}$ & 75.84 & 1.09 & 5.45 & 17.62 \\
GAL $1.0 \mu \mathrm{mol} / \mathrm{L}$ & 68.92 & 2.17 & 5.95 & 22.95 \\
GAL $2.0 \mu \mathrm{mol} / \mathrm{L}$ & 47.62 & 9.14 & 5.90 & 37.35 \\
\hline
\end{tabular}

$(0,0.5,1$, and $2 \mu \mathrm{mol} / \mathrm{L})$ for $24 \mathrm{~h}$ was visualized using Hoechst 33258 staining. MCF-7 cells were plated in 6-well plates at a density of $5 \times 10^{4}$ cells per well and incubated with GAL. After $24 \mathrm{~h}$, the cells were incubated with Hoechst $33258(5 \mu \mathrm{g} / \mathrm{mL})$ for $30 \mathrm{~min}$ at room temperature. Following washing with PBS, the cells were visualized and photographed under an Olympus inverted fluorescence microscope (Tokyo, Japan).

2.6. Flow Cytometric Analysis of Cell Apoptosis. Apoptosis was determined using an annexin V-FITC apoptosis kit (BD Pharmingen, Franklin Lakes, NJ, USA) according to manufacturer's instructions. After treatment with GAL (0, $0.5,1$, and $2 \mu \mathrm{mol} / \mathrm{L})$ for $24 \mathrm{~h}$, cells were washed with icecold PBS and resuspended in binding buffer $(10 \mathrm{mmol} / \mathrm{L}$ HEPES, pH 7.4, $140 \mathrm{mmol} / \mathrm{L} \mathrm{NaCl}$, and $2.5 \mathrm{mmol} / \mathrm{L} \mathrm{CaCl}_{2}$ ) at a concentration of $1 \times 10^{6}$ cells $/ \mathrm{mL}$. Cells were stained with annexin V-FITC and propidium (PI) for $15 \mathrm{~min}$ in dark before being analyzed with flow cytometer (Beckman Coulter Inc., Miami, FL, USA).

2.7. Western Blotting Analysis. MCF-7 cells were treated with various concentrations of GAL $(0,0.25,0.5,1$, and $2 \mu \mathrm{mol} / \mathrm{L})$ for $24 \mathrm{~h}$ in $25 \mathrm{~cm}^{2}$ flask. The cells were collected, washed twice with PBS, and then lysed with RIPA buffer and protease and phosphatase inhibitors cocktail (Roche, Beijing, China) for 20 min on ice. The cell lysates were cleared by centrifugation at $12,000 \mathrm{~g}$ for $20 \mathrm{~min}$. Protein concentrations were determined by Bradford assay. Equal amounts of lysate $(40 \mu \mathrm{g})$ were resolved by SDS-PAGE and transferred to polyvinylidene difluoride membrane (Millipore Corp., Bedford, MA, USA). Membranes were blocked in TBST containing 5\% nonfat skim milk at room temperature for $2 \mathrm{~h}$ and probed with primary antibodies overnight at $4^{\circ} \mathrm{C}$. Then membranes were blotted with an appropriate horseradish peroxidase-linked secondary antibody. Proteins were visualized using enhanced chemiluminescence Western blotting detection reagents (Amersham Pharmacia Biotech, Inc., Piscataway, NJ, USA).

2.8. RNA Interference. FOXO3a (FKHRL1) small interfering RNA (siRNA) and the scrambled siRNA were purchased from Santa Cruz Technology (Dallas, TX, USA). After FOXO3a siRNA or NC siRNA was transfected into MCF-7 cells using silMPORTER (Upstate, Virginia, USA) for $24 \mathrm{~h}$, $1 \mu \mathrm{mol} / \mathrm{L}$ GAL was added for $24 \mathrm{~h}$. After GAL treatment for $24 \mathrm{~h}$, the cells were harvested. Then, cells apoptosis was checked through Hoechst staining and protein expression correlated with apoptosis was detected by Western blotting analysis.
2.9. Statistical Analysis. Data are presented as mean \pm standard deviation (SD). Statistical analysis was performed using SPSS 11.5 software (SPSS Inc., Chicago, Illinois, USA). Comparison between groups was performed with Student's $t$-test. A $P$ value of $\leq 0.05$ was considered statistically significant.

\section{Results}

3.1. The Molecular Structure and the Identification of GAL. The molecular weight of GAL is 775 and molecular formula is $\mathrm{C}_{44} \mathrm{H}_{58} \mathrm{~N}_{2} \mathrm{O}_{10}$ (Figure 1(a)). GAL is determined by MatrixAssisted Laser Desorption/Ionization Time of Flight Mass Spectrometry and the peak value is 755.4 Da (Figure 1(b)).

3.2. GAL Inhibited the Proliferation of MCF-7 Cells and NAC Could Block Its Inhibition. The proliferation inhibitory effect of GAL in human MCF-7 cells was examined with MTT assay as described in Section 2. Cells were cultured for $48 \mathrm{~h}$ (Figure 2(a)) in the presence of various concentrations of GAL. MCF-7 cells showed a decreased cell proliferation after treatment with GAL. The $\mathrm{IC}_{50}$ value of GAL for MCF-7 cells is $1.46 \mu \mathrm{mol} / \mathrm{L}$ comparable with GA $\left(\mathrm{IC}_{50}, 1.16 \mu \mathrm{mol} / \mathrm{L}\right)$. Cells were cultured for different times $(0,6,12,24,36,48$, and $60 \mathrm{~h}$ ) in the presence of $2 \mu \mathrm{mol} / \mathrm{L}$ GAL. MCF-7 cells showed a decreased cell proliferation over time (Figure 2(b)). In addition, the proliferation inhibitory effect of GAL in human MCF-7 cells was blocked, when NAC $(0,1,2$, and $4 \mathrm{mmol} / \mathrm{L})$ was added in combination with GAL (Figure 2(c)).

3.3. Induction of Oxidative Stress in MCF-7 Cells. The intracellular ROS level was stained with DCFH-DA and determined by fluorescence microscopy and fluorescence spectrophotometer as described in Section 2. GAL could increase ROS level in dose dependent manner; however NAC could decrease the increase of ROS induced by GAL (Figure 3).

3.4. Induction of Apoptosis by GAL in MCF-7 Cells. By Hoechst 33258 staining the nuclei of untreated cells were normal in appearance and showed diffused staining of the chromatin. After exposure to GAL for $24 \mathrm{~h}$, most cells presented typical morphological changes of apoptosis such as chromatin condensation, cell shrinkage, chromatin margination, or apoptotic bodies (Figure 4(a)). Induction of apoptosis by GAL was further confirmed by annexin V-FITC/PI staining. GAL $(0.5,1,2 \mu \mathrm{mol} / \mathrm{L})$ induced apoptosis in MCF7 cells in dose dependent manner and the ratio of apoptosis was $23.07 \%, 28.9 \%$, and $43.25 \%$, respectively (Figure $4(\mathrm{~b})$, Table 1). It suggested that apoptosis was the predominant mode of GAL-induced cell death. 


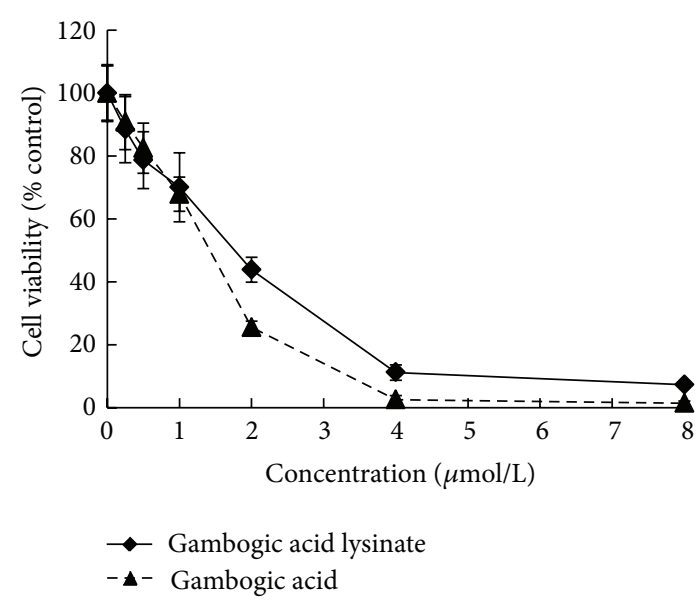

(a)

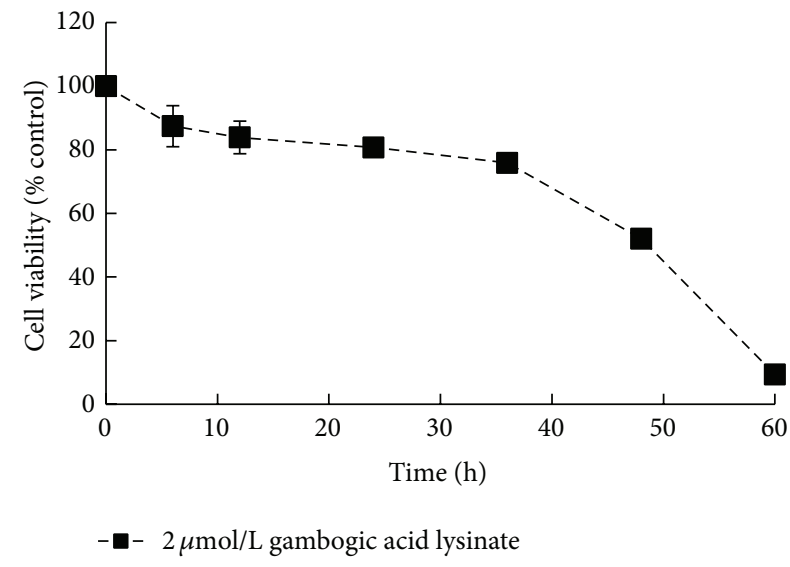

(b)

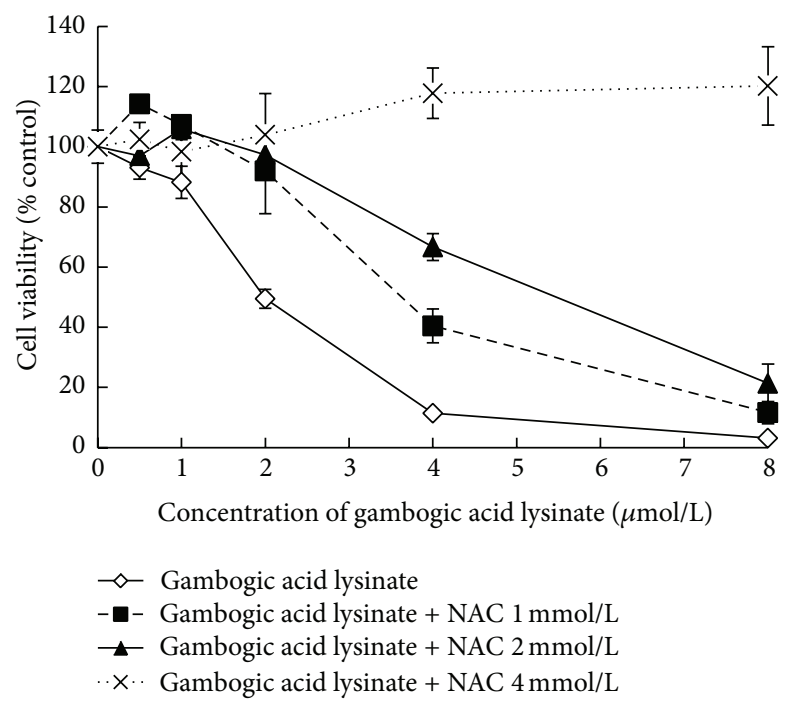

(c)

FIGURE 2: Gambogic acid lysinate can inhibit MCF-7 cell proliferation; however NAC can block it. (a) Exponential growth MCF-7 cells were treated with various concentrations of gambogic acid lysinate $(0,0.25,0.5,1,2,4$, and $8 \mu \mathrm{mol} / \mathrm{L}$ ) for $48 \mathrm{~h}$; (b) exponential growth MCF-7 cells were treated with $2 \mu \mathrm{mol} / \mathrm{L}$ gambogic acid lysinate for different times $(0,6,12,24,36,48$, and $60 \mathrm{~h})$; (c) exponential growth MCF-7 cells were treated with various concentrations of gambogic acid lysinate $(0,0.25,0.5,1,2,4$, and $8 \mu \mathrm{mol} / \mathrm{L})$ plus various concentrations of NAC $(0,1,2$, and $4 \mathrm{mmol} / \mathrm{L}$ ) for $48 \mathrm{~h}$, and cell viability was detected by MTT method.

3.5. Downregulation of SIRT1 and Upregulation of C-Caspase3 in GAL-Induced Apoptosis in MCF-7 Cells. In order to investigate the role of apoptosis, the activity of SIRT1 and caspase-3 in response to GAL treatment was determined. SIRT1 expression was decreased by GAL in a dose dependent manner. However, the phosphorylation of FOXO3a and the expression p27Kip1 were increased by GAL in a dose dependent manner. In addition the expression of cleaved caspase3 also increased in a dose dependent manner; however the expression of caspase- 3 decreased at GAL $2 \mu \mathrm{mol} / \mathrm{L}$ treated group (Figure 5). There is no change of the expression of $\mathrm{Bcl}-$ 2 and Bax.

3.6. FOXO3a siRNA Partly Blocked Cell Apoptosis Induced by GAL in MCF-7 Cells. Compared with control group, after exposure to GAL+NC siRNA for $24 \mathrm{~h}$, most cells presented typical morphological changes of apoptosis such as chromatin condensation, cell shrinkage, chromatin margination, or apoptotic bodies. Compared with GAL+NC siRNA group, GAL+FOXO3a siRNA group partly blocked the effect of GAL (Figure 6(a)). By Western blot analysis we found that FOXO3a siRNA decreased the expression of FOXO3, the phosphorylation of FOXO3, and the expression of p27Kip1, but with no effect on the expression of SIRT1, caspase-3, Bax, and Bcl-2 (Figure 6(b)).

\section{Discussion and Conclusions}

Breast cancer is the most common cancer among women in China and western countries. It is also the principal cause of cancer death for females. As a result of "westernized lifestyles" and exogenous estrogen exposure, there is an increasing trend 

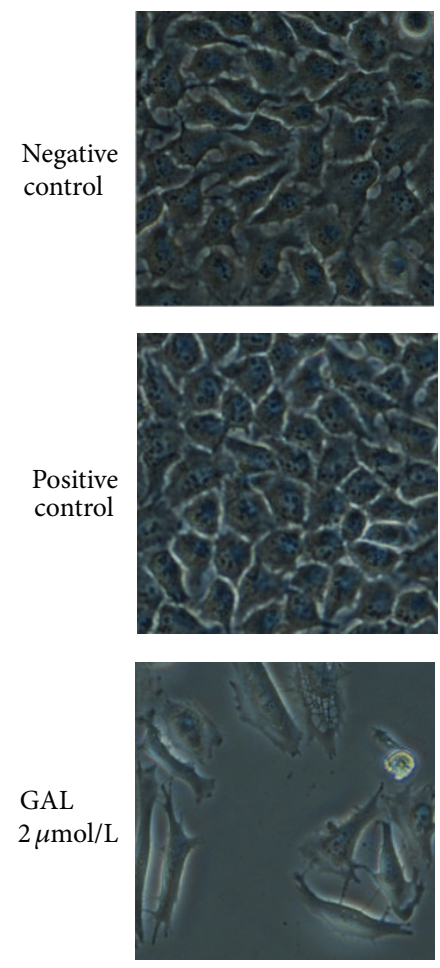

Light
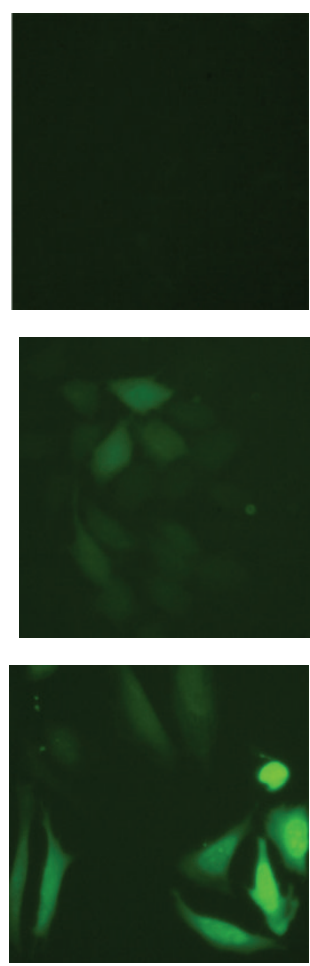

Fluorescence
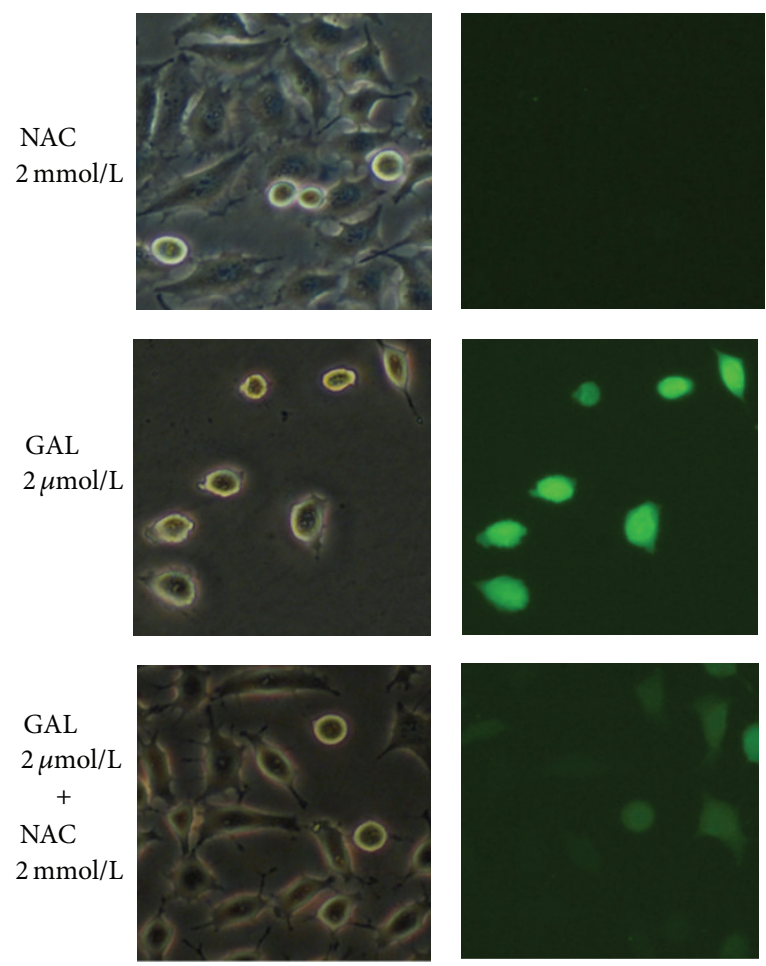

Light

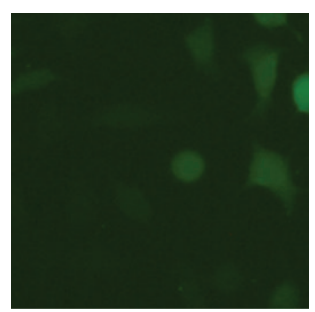

Fluorescence

(a)

(b)

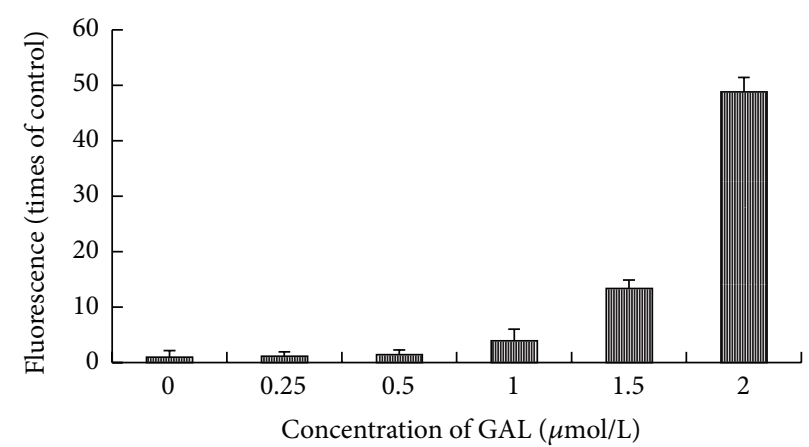

(c)

FIGURE 3: Gambogic acid lysinate can increase intracellular ROS level and NAC can block it. Exponential growth MCF-7 cells were treated with PBS (negative control), Rosup $100 \mathrm{mg} / \mathrm{L}$ (positive control), gambogic acid lysinate $2 \mu \mathrm{mol} / \mathrm{L}$, NAC $2 \mathrm{mmol} / \mathrm{L}$, and gambogic acid lysinate $2 \mu \mathrm{mol} / \mathrm{L}+\mathrm{NAC} 2 \mathrm{mmol} / \mathrm{L}$ for $24 \mathrm{~h}$. After treatment MCF-7 cells were stained with DCFH-DA and were determined by fluorescence microscopy (a, b) and fluorescence spectrophotometer (c).

of breast cancer incidence in China in the latest decades $[1,12]$. SIRT1 physiologically interacts with p53 and attenuates its functions through deacetylation at its C-terminal Lys382 residue [13]. The expression of SIRT1 protein was seen in most human breast cancer specimens, and its expression was significantly associated with distant metastasis and poor prognosis [14-16] and reported to function as a tumor promoter. Recently, SIRT1 has emerged as a potent therapeutic target to cancer treatment. Therefore, identification of potent and unique SIRT1 inhibitor for cancer treatment is urgently needed.

GAL is the salt of lysine and gambogic acid, which has antitumor activities in a broad range of human cancer cells
[17]. The difference between GAL and GA is that GAL can dissolve in water; however GA cannot. In this study, we observed that the inhibition of GAL to MCF-7 cell proliferation was comparable to that of $\mathrm{GA}$ and the $\mathrm{IC}_{50}$ values of $\mathrm{GAL}$ and GA were 1.46 and $1.16 \mu \mathrm{mol} / \mathrm{L}$, respectively. GAL could inhibit MCF-7 cell proliferation in dose and time dependent manner (Figures 2(a) and 2(b)). We also found that NAC can block the activity of GAL, when GAL was administrated in combination with NAC $(1,2$, and $4 \mathrm{mmol} / \mathrm{L})$. The block of NAC to the activity of GAL was increased with the increase of the concentration of NAC (Figure 2(c)). It is well known that NAC is an ROS scavenger and is frequently used as a precursor to GSH, which supports the synthesis of GSH, and 


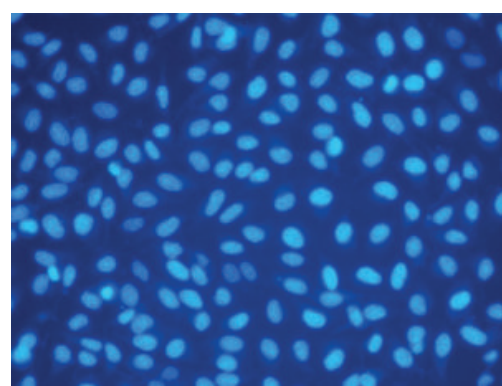

Control

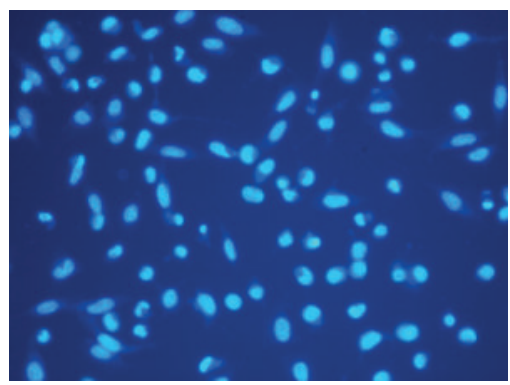

GAL $1.0 \mu \mathrm{mol} / \mathrm{L}$

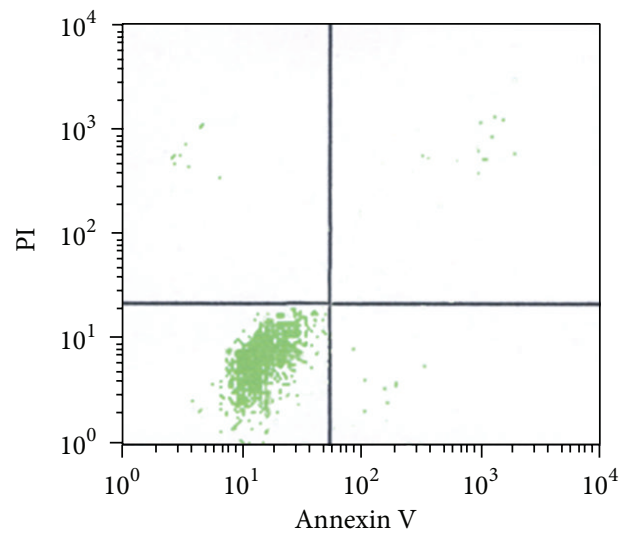

Control

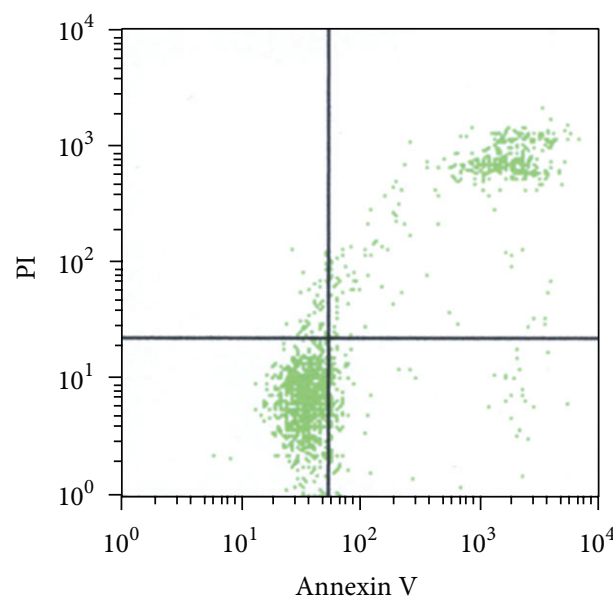

GAL $1.0 \mu \mathrm{mol} / \mathrm{L}$

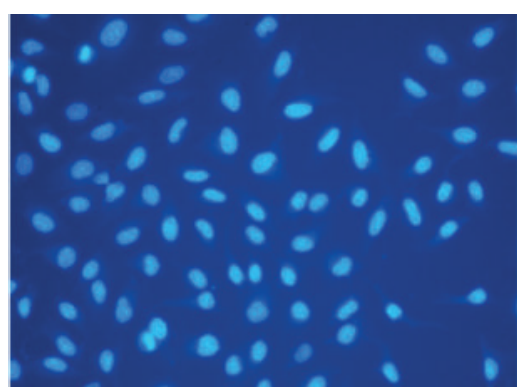

GAL $0.5 \mu \mathrm{mol} / \mathrm{L}$

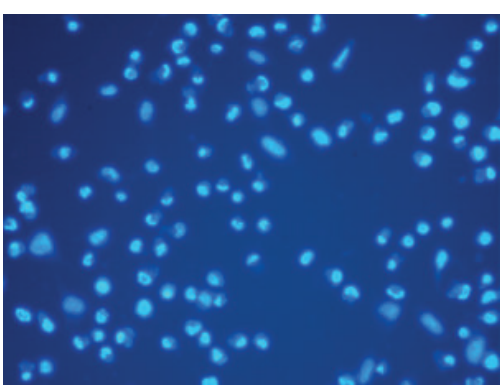

GAL $2.0 \mu \mathrm{mol} / \mathrm{L}$

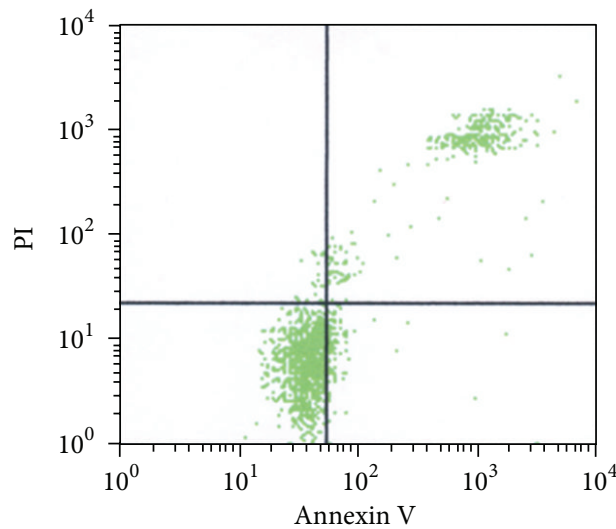

GAL $0.5 \mu \mathrm{mol} / \mathrm{L}$

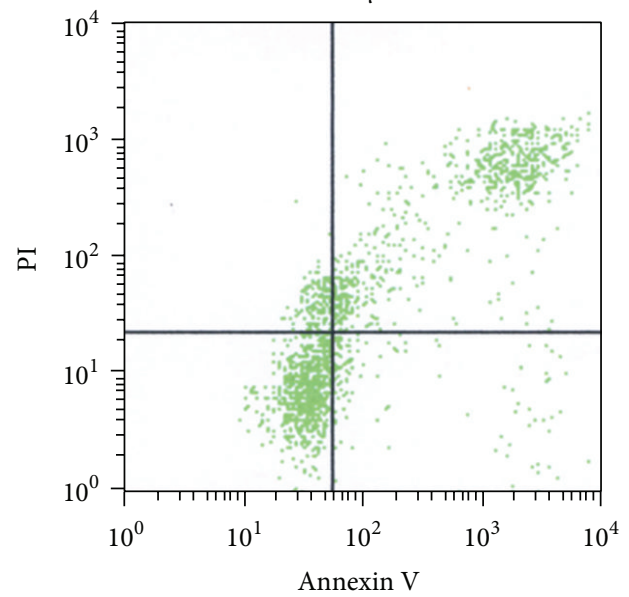

GAL $2.0 \mu \mathrm{mol} / \mathrm{L}$

(b)

FIGURE 4: Gambogic acid lysinate can induce MCF-7 cell apoptosis. Exponential growth MCF-7 cells were treated with GAL (0, 0.5, 1, 1.5, and $2 \mu \mathrm{mol} / \mathrm{L}$ ) for $24 \mathrm{~h}$. (a) After treatment MCF-7 cells were stained with Hoechst 33258 and observed by fluorescence microscope. (b) After treatment MCF-7 cells were stained with annexin V-FITC and PI and analyzed with flow cytometer. 

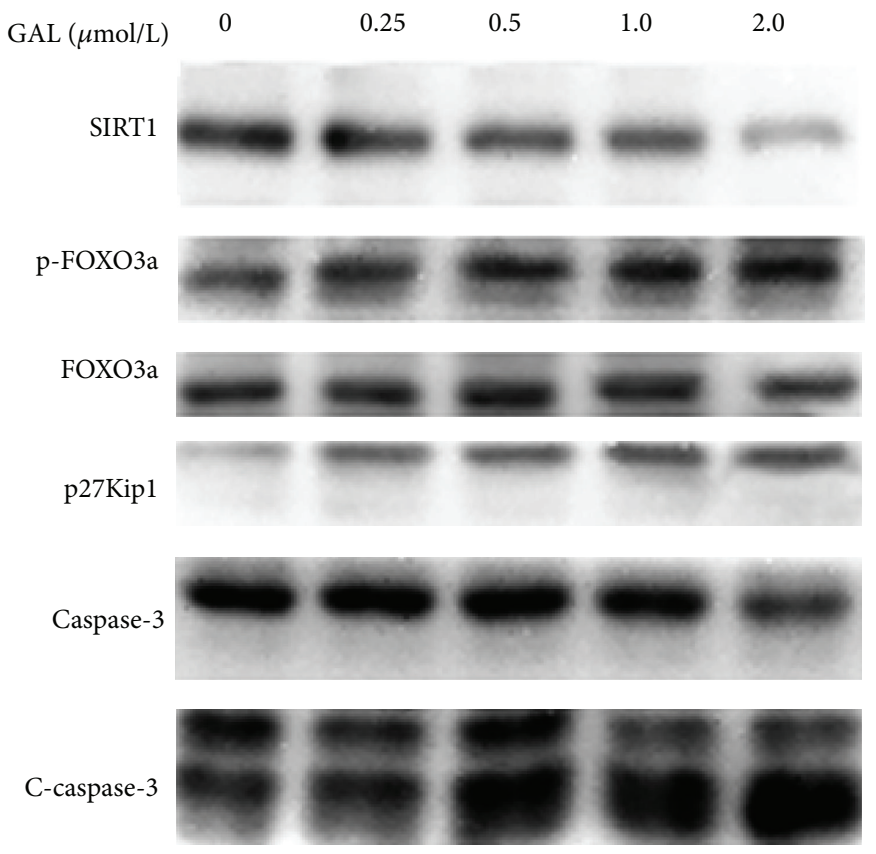

Bax

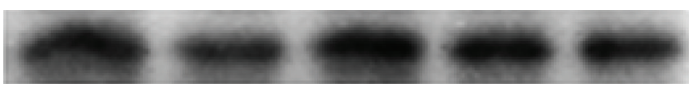

$\mathrm{Bcl}-2$

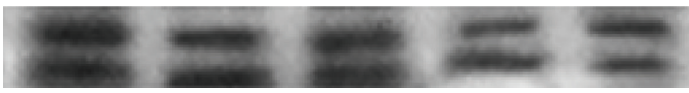

$\beta$-actin
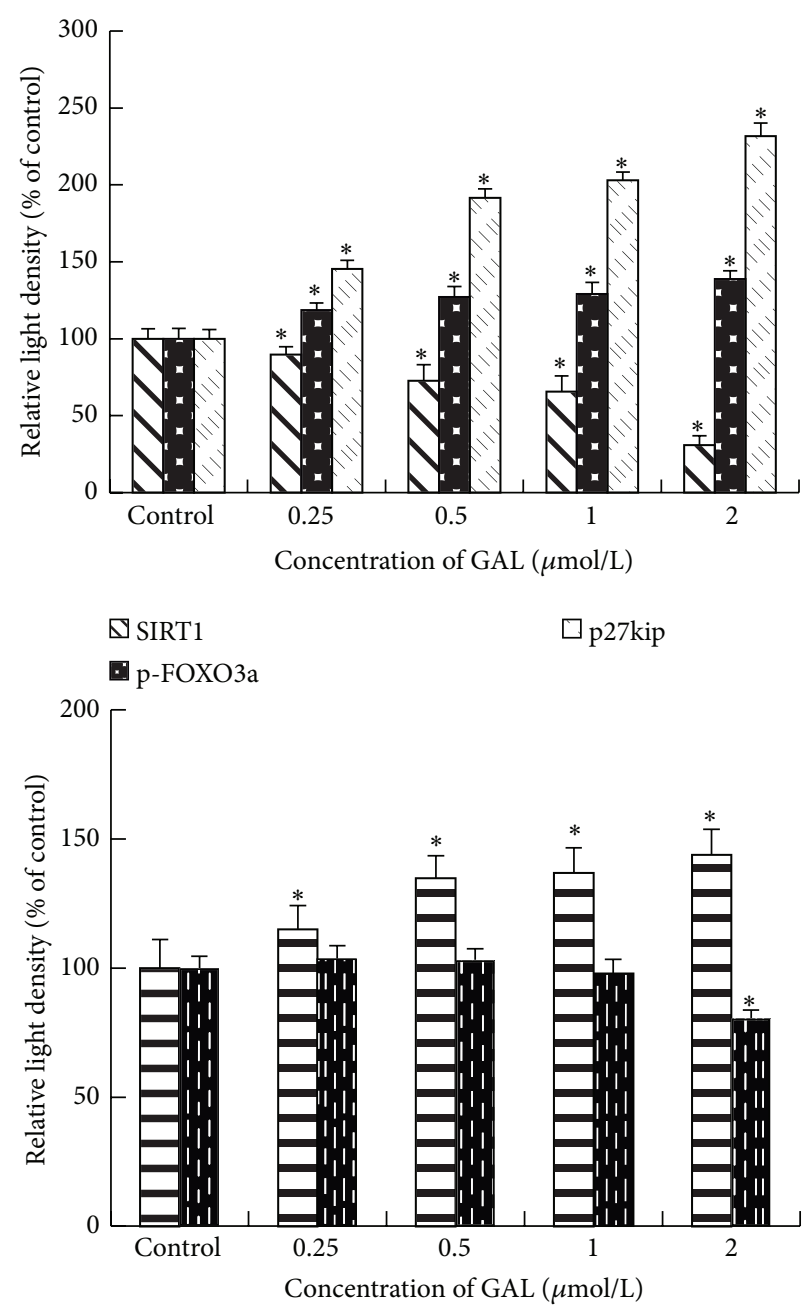

目 C-caspase-3 I Caspase-3

FIGURE 5: Gambogic acid lysinate can induce MCF-7 cells apoptosis by SIRT1 and caspase-3 signal pathway. MCF-7 cells were treated with the indicated concentrations of GAL for $24 \mathrm{~h}$, and the cell extracts were prepared and analyzed by Western blotting with corresponding antibodies. The blots were quantified by densitometry with Scion Image (Scion Corporation, Frederick, MD, USA), and the relative ratio of target protein to $\beta$-actin was calculated and expressed as the mean \pm SD from three experiments. ${ }^{*} P<0.05$ versus the control group.

assists in replenishing GSH when stores are compromised during oxidative or electrophilic stress $[18,19]$. It can be deduced that GAL can inhibit MCF-7 cell proliferation by increasing ROS level, and NAC can remove the ROS induced by GAL. It is further confirmed by fluorescence microscope and fluorescence spectrophotometer by which intracellular ROS can be determined.

It is well known that ROS can induce cell apoptosis by various signal pathways including targeting cytosolic thioredoxin reductase [20], JNK/ATF2 pathway [21], p53mitochondrial pathway [22], and AMPK/SIRT1/PGC-1 $\alpha$ signal pathway [23]. It has been reported that GA has the ability to activate apoptotic signaling in numerous types of cancer cells. Herein, we also demonstrated that GAL kills MCF-7 cells predominantly through induction of apoptosis (Figure 4). GAL triggers apoptotic cell death in dose dependent manner. In Western blot analysis we also observed that
SIRT1 expression was decreased in dose dependent manner. SIRT1 is one of the cytoplasmic $\mathrm{NAD}^{+}$-dependent histone deacetylases and deacetylates histone $\mathrm{H} 3$ lysine 56 (H3K56) and a-tubulin. It also shares nonhistone substrates of FOXO1, FOXO3, and p53 with SIRT1 [24]. In previous study it is reported that forkhead transcription factors can be inhibited by the deacetylase SIRT1 [25], and increased expression of any FOXO member results in the activation of the cell cycle inhibitor p27Kip1 [26]. In this study we investigated the change of FOXO3a and p27Kipl. We found that the phosphorylation of FOXO3a and the expression of p27Kipl increased with the decrease of SIRT1 expression. It can be concluded that SIRT1/FOXO3a/p27Kip1 signal pathway is one of the pathways by which GAL induced MCF-7 cell apoptosis. In the meantime we also observed that GAL enhanced the expression of cleaved caspase- 3 . It can be inferred that caspase- 3 signal pathway also takes part in the apoptotic process induced 


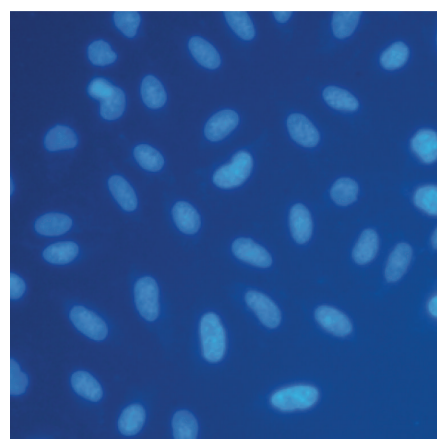

Control

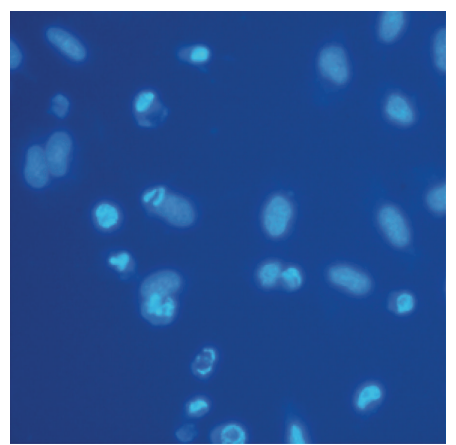

NC siRNA

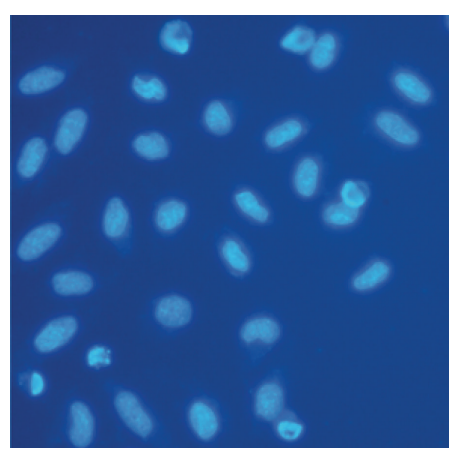

FOXO3a siRNA

(a)
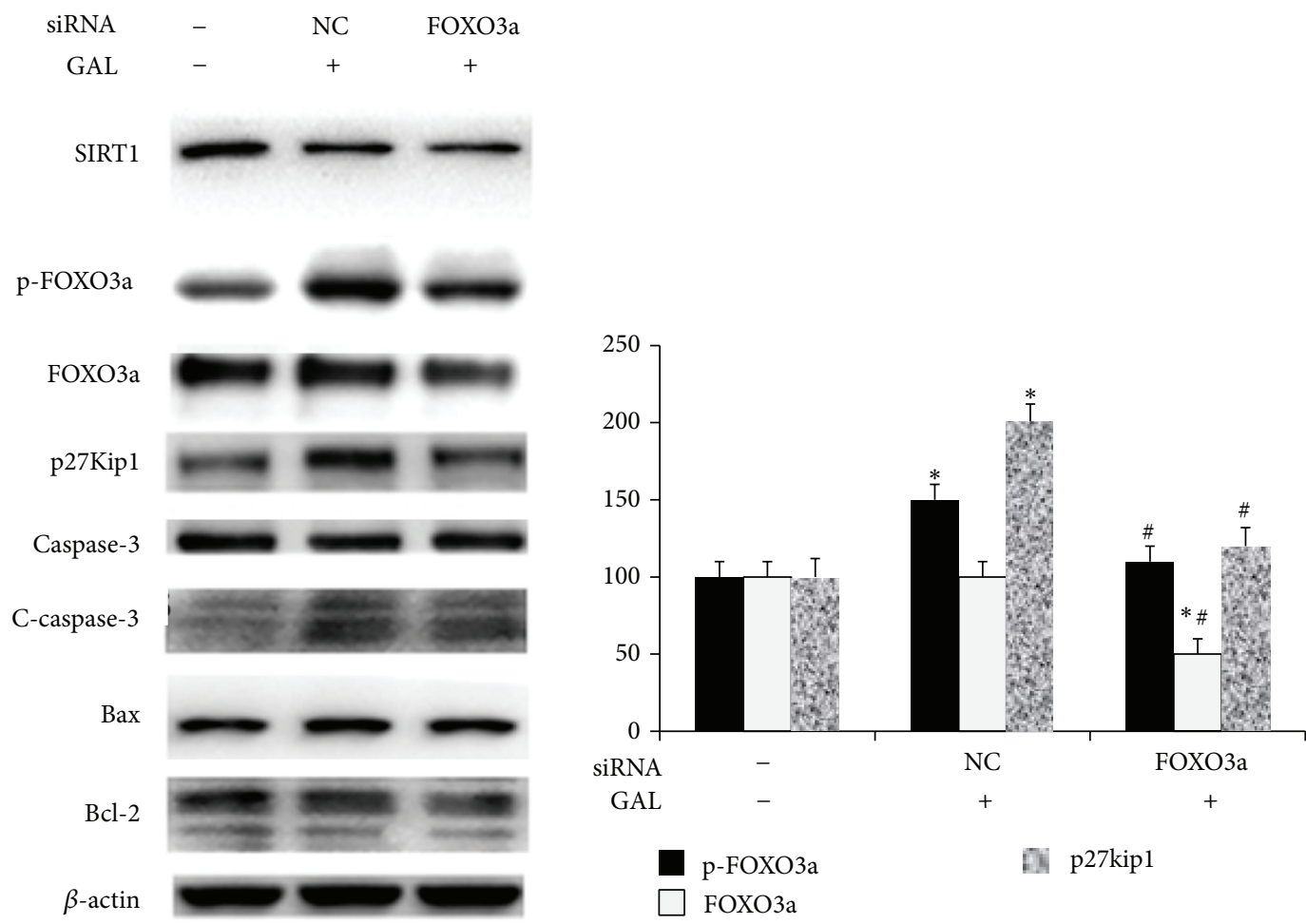

(b)

FIGURE 6: FOXO3a siRNA partly blocked cell apoptosis induced by GAL in MCF-7 cells. MCF-7 cells were transfected with NC or FOXO3a siRNA for $24 \mathrm{~h}$; then $1 \mu \mathrm{mol} / \mathrm{L}$ GAL was added for $24 \mathrm{~h}$. After GAL treatment for $24 \mathrm{~h}$, (a) MCF-7 cells were stained with Hoechst 33258 and observed by fluorescence microscope; (b) MCF-7 cells were harvested and cell extracts were prepared and analyzed by Western blotting with corresponding antibodies. The blots were quantified by densitometry with Scion Image (Scion Corporation, Frederick, MD, USA), and the relative ratio of target protein to $\beta$-actin was calculated and expressed as the mean $\pm \mathrm{SD}$ from three experiments. ${ }^{*} P<0.05$ versus the control group; ${ }^{\#} P<0.05$ versus the GAL+NC siRNA group.

by GAL. At knockdown of FOXO3a, we found the expression of FOXO3, the phosphorylation of FOXO3, and the expression of p27Kipl were decreased and cell apoptosis induced by GAL was partly blocked; however the expression of SIRT1, caspase-3, Bax, and Bcl-2 did not change (Figure 6(b)). It was further confirmed that SIRT1/FOXO3a/p27Kip1 signal pathway is only one of the pathways by which GAL induced MCF-7 cell apoptosis.

In conclusion these results suggested that GAL inhibited MCF-7 cells proliferation and induced MCF-7 cells apoptosis by increasing ROS level which could induce cell apoptosis by both SIRT1/FOXO3a/p27Kip1 and caspase-3 signal pathway. GAL may be a new chemotherapy drug for breast cancer.

\section{Conflict of Interests}

The authors declare that they have no conflict of interests regarding the publication of this paper. 


\section{Authors' Contribution}

Yong-Zhan Zhen and Ya-Jun Lin contributed equally to this work.

\section{Acknowledgment}

This work was financially supported by a grant from the National Natural Science Foundation of China (no. 81001439).

\section{References}

[1] C. Desantis, J. Ma, L. Bryan, and A. Jemal, "Breast cancer statistics, 2013," CA Cancer Journal for Clinicians, vol. 64, no. 1, pp. 52-62, 2014.

[2] G. Fountzilas, U. Dafni, C. Papadimitriou et al., "Dose-dense sequential adjuvant chemotherapy followed, as indicated, by trastuzumab for one year in patients with early breast cancer: first report at 5-year median follow-up of a Hellenic Cooperative Oncology Group randomized phase III trial," BMC Cancer, vol. 14, no. 1, p. 515, 2014.

[3] L. Amiri-Kordestani, G. M. Blumenthal, Q. C. Xu et al., "FDA approval: ado-trastuzumab emtansine for the treatment of patients with HER2-positive metastatic breast cancer," Clinical Cancer Research, vol. 20, no. 17, pp. 4436-4441, 2014.

[4] H. Wang, T. Vo, A. Hajar et al., "Multiple mechanisms underlying acquired resistance to taxanes in selected docetaxel-resistant MCF-7 breast cancer cells," BMC Cancer, vol. 14, no. 1, article 37, 2014.

[5] X. Wang and W. Chen, "Gambogic acid is a novel anticancer agent that inhibits cell proliferation, angiogenesis and metastasis," Anti-Cancer Agents in Medicinal Chemistry, vol. 12, no. 8, pp. 994-1000, 2012.

[6] R. Cascão, B. Vidal, H. Raquel et al., "Potent anti-inflammatory and antiproliferative effects of gambogic acid in a rat model of antigen-induced arthritis," Mediators of Inflammation, vol. 2014, Article ID 195327, 7 pages, 2014.

[7] J. Wen, H. Pei, X. Wang et al., "Gambogic acid exhibits anti-psoriatic efficacy through inhibition of angiogenesis and inflammation," Journal of Dermatological Science, vol. 74, no. 3, pp. 242-250, 2014.

[8] Q. Qi, N. Lu, C. Li et al., "Involvement of RECK in gambogic acid induced anti-invasive effect in A549 human lung carcinoma cells," Molecular Carcinogenesis, 2014.

[9] N. Lu, H. Hui, H. Yang et al., "Gambogic acid inhibits angiogenesis through inhibiting PHD2-VHL-HIF- $1 \alpha$ pathway," European Journal of Pharmaceutical Sciences, vol. 49, no. 2, pp. 220-226, 2013.

[10] D. Duan, B. Zhang, J. Yao et al., "Gambogic acid induces apoptosis in hepatocellular carcinoma SMMC-7721 cells by targeting cytosolic thioredoxin reductase," Free Radical Biology and Medicine, vol. 69, pp. 15-25, 2014.

[11] H. Zhang, Y. Lei, P. Yuan et al., "ROS-mediated autophagy induced by dysregulation of lipid metabolism plays a protective role in colorectal cancer cells treated with gambogic acid," PLoS ONE, vol. 9, no. 5, Article ID e96418, 2014.

[12] H. Zeng, R. Zheng, S. Zhang, X. Zou, and W. Chen, "Female breast cancer statistics of 2010 in China: estimates based on data from 145 population-based cancer registries," Journal of Thoracic Disease, vol. 6, no. 5, pp. 466-470, 2014.
[13] L. R. Saunders and E. Verdin, "Sirtuins: critical regulators at the crossroads between cancer and aging," Oncogene, vol. 26, no. 37, pp. 5489-5504, 2007.

[14] S. Proietti, A. Cucina, G. Dobrowolny et al., "Melatonin downregulates MDM2 gene expression and enhances p53 acetylation in MCF-7 cells," Journal of Pineal Research, vol. 57, no. 1, pp. 120129, 2014.

[15] B. J. Hwang, A. Madabushi, J. Jin, S. Y. Lin, and A. L. Lu, "Histone/protein deacetylase SIRT1 is an anticancer therapeutic target," American Journal of Cancer Research, vol. 4, no. 3, pp. 211-221, 2014.

[16] H. Yuan, L. Su, and W. Y. Chen, "The emerging and diverse roles of sirtuins in cancer: a clinical perspective," OncoTargets and Therapy, vol. 6, pp. 1399-1416, 2013.

[17] X. Shi, X. Chen, X. Li et al., "Gambogic acid induces apoptosis in imatinib-resistant chronic myeloid leukemia cells via inducing proteasome inhibition and caspase-dependent bcr-abl downregulation AC," Clinical Cancer Research, vol. 20, no. 1, pp. 151163,2014

[18] I. Downs, J. Liu, T. Y. Aw, P. A. Adegboyega, and M. N. Ajuebor, "The ROS scavenger, NAC, regulates hepatic V $\alpha 14$ iNKT cells signaling during Fas mAB-dependent fulminant liver failure," PLoS ONE, vol. 7, no. 6, Article ID e38051, 2012.

[19] F. Zhang, S. S. Lau, and T. J. Monks, "The cytoprotective effect of N-acetyl-L-cysteine against ROS-induced cytotoxicity is independent of its ability to enhance glutathione synthesis," Toxicological Sciences, vol. 120, no. 1, pp. 87-97, 2011.

[20] D. Duan, B. Zhang, J. Yao, Y. Liu, and J. Fang, "Shikonin targets cytosolic thioredoxin reductase to induce ROS-mediated apoptosis in human promyelocytic leukemia HL-60 cells," Free Radical Biology and Medicine, vol. 70, pp. 182-193, 2014.

[21] J. J. An, K. J. Shi, W. Wei et al., “The ROS/JNK/ATF2 pathway mediates seleniteinduced leukemia NB4 cell cycle arrest and apoptosis in vitro and in vivo," Cell Death and Disease, vol. 4, no. 12, article e973, 2013.

[22] M.-J. Xie, Y.-H. Ma, L. Miao et al., "Emodin-provoked oxidative stress induces apoptosis in human colon cancer HCT116 cells through a p53-mitochondrial apoptotic pathway," Asian Pacific Journal of Cancer Prevention, vol. 15, no. 13, pp. 5201-5205, 2014.

[23] B. Huang, X. Cheng, D. Wang et al., "Adiponectin promotes pancreatic cancer progression by inhibiting apoptosis via the activation of AMPK/Sirt1/PGC-1 $\alpha$ signaling," Oncotarget, vol. 5, no. 13, pp. 4732-4745, 2014.

[24] S. Hirai, S. Endo, R. Saito et al., "Antitumor effects of a sirtuin inhibitor, tenovin-6, against gastric cancer cells via death receptor 5 up-regulation," PLOS ONE, vol. 9, no. 7, Article ID e102831, 2014.

[25] Y. Yang, H. Hou, E. M. Haller, S. V. Nicosia, and W. Bai, "Suppression of FOXO1 activity by FHL2 through SIRT1mediated deacetylation," The EMBO Journal, vol. 24, no. 5, pp. 1021-1032, 2005.

[26] G.-Z. Tao, N. Lehwald, K. Y. Jang et al., "Wnt/ $\beta$-catenin signaling protects mouse liver against oxidative stress-induced apoptosis through the inhibition of forkhead transcription factor $\mathrm{FoxO}_{3}$," The Journal of Biological Chemistry, vol. 288, no. 24, pp. 17214-17224, 2013. 


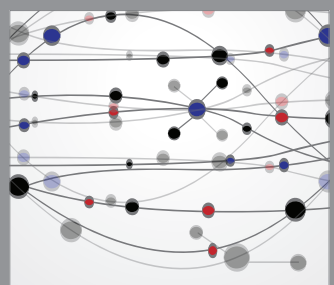

The Scientific World Journal
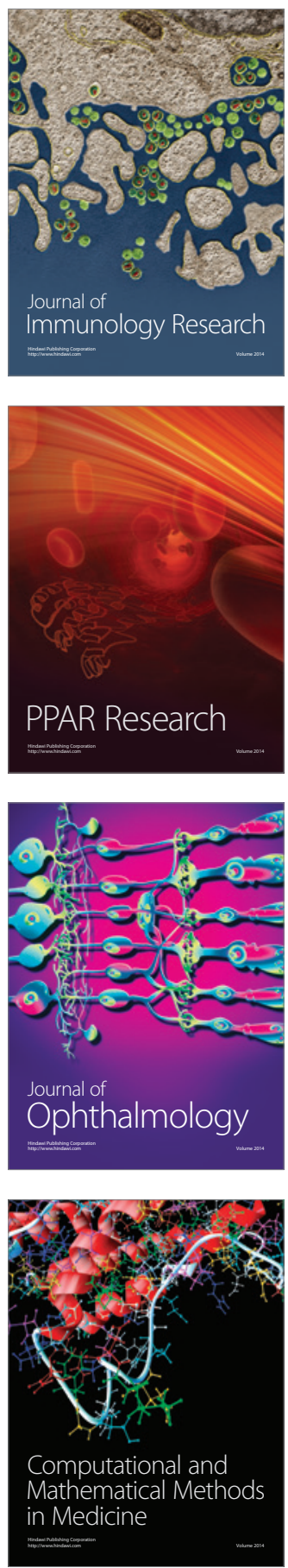

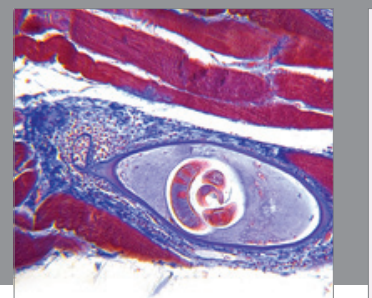

Gastroenterology

Research and Practice
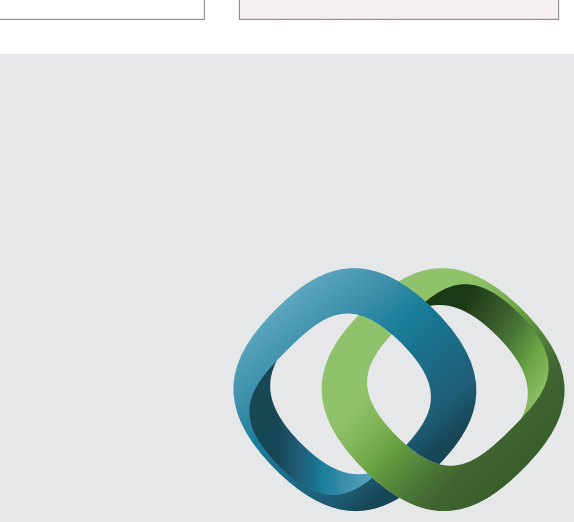

\section{Hindawi}

Submit your manuscripts at

http://www.hindawi.com
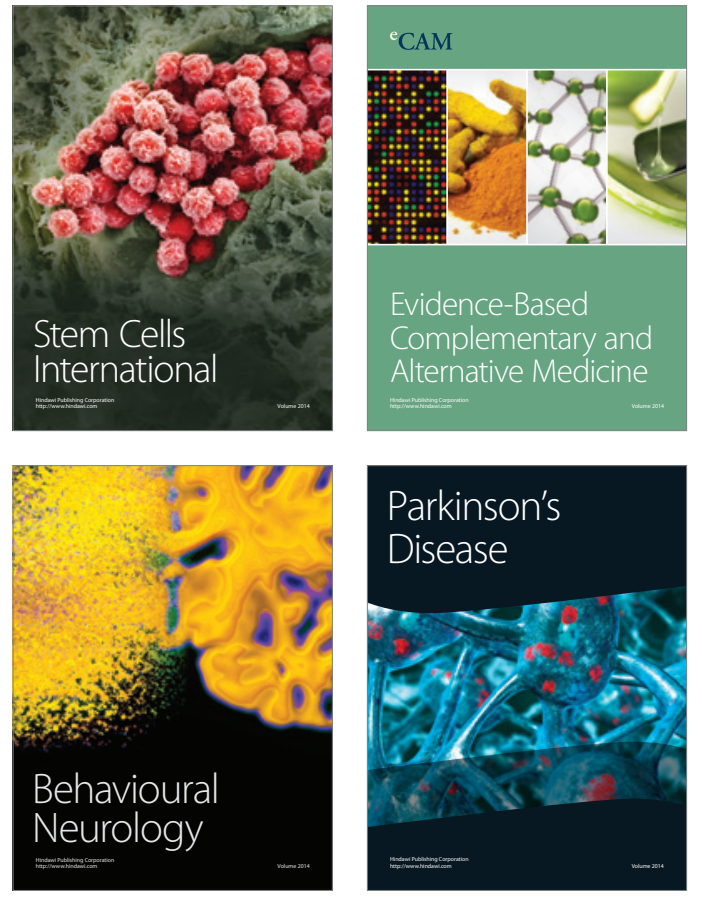
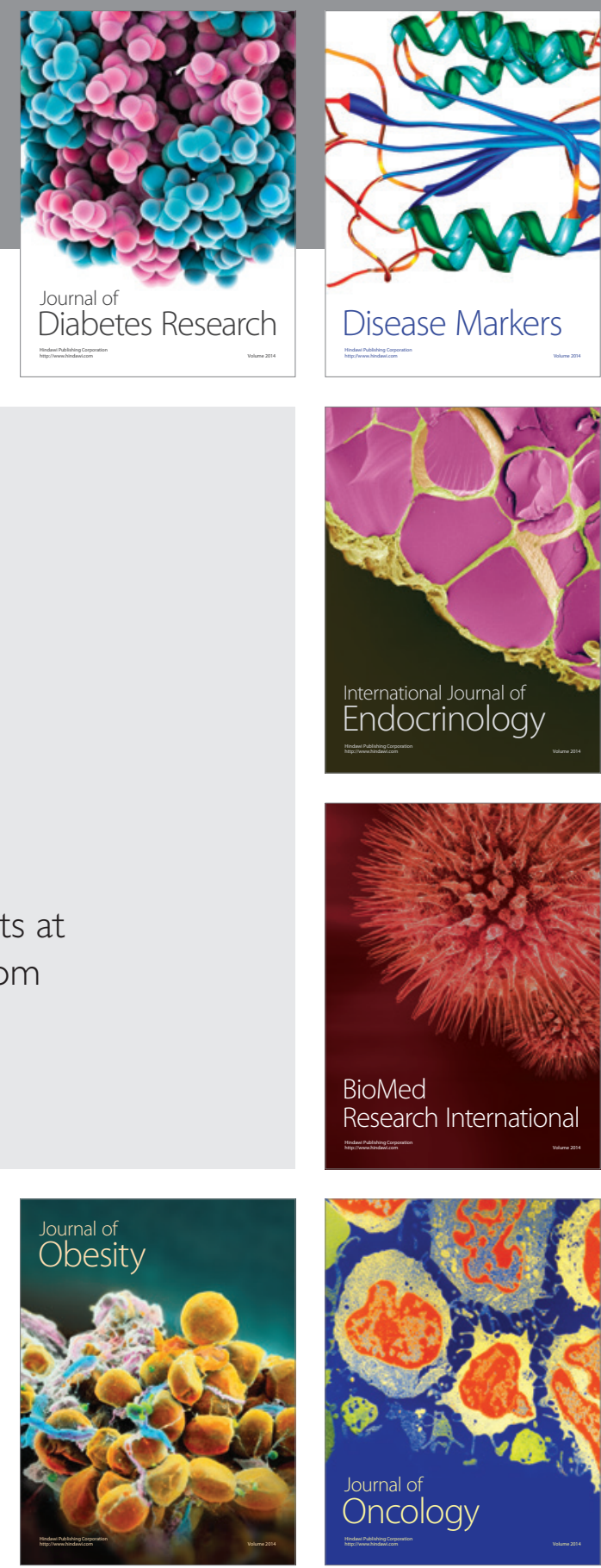

Disease Markers
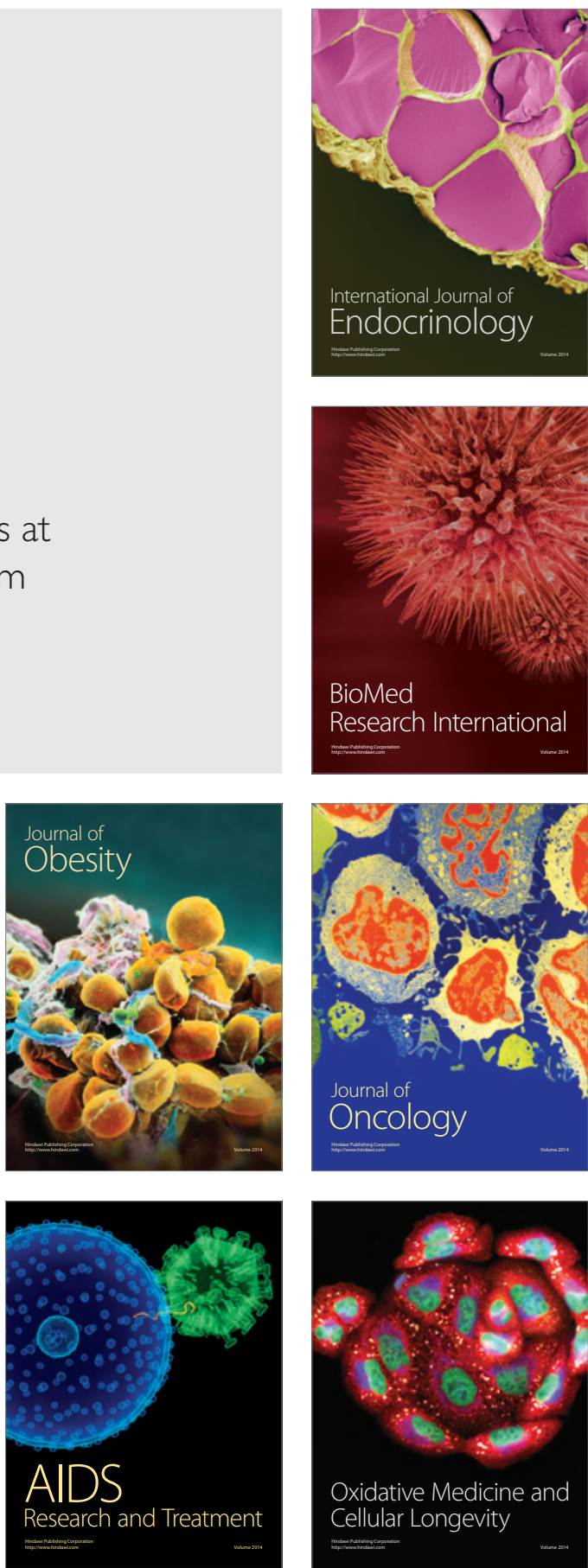